\section{Correction: Gender Differences in the Inheritance Mode of RYR2 Mutations in Catecholaminergic Polymorphic Ventricular Tachycardia Patients}

\author{
Seiko Ohno, Kanae Hasegawa, Minoru Horie
}

In the Abstract, there is an error in the sixth sentence. The correct sentence is: The inheritance of RYR2 mutations was significantly more frequent from mothers $(\mathrm{n}=12,34.3 \%)$ than fathers $(\mathrm{n}=2,7.4 \%)(\mathrm{P}=0.015)$. In the Origin of the mutations subsection of the Results, there is a similar error in the second sentence of the second paragraph. The correct sentence is: The frequency of mutations originating from mothers was significantly higher than that from fathers $(\mathrm{P}=0.015)$.

There are several errors in the Location of mutations subsection of the Results. The correct paragraph is: Among 12 mutations inherited from mothers, seven (58.3\%) were located in the $\mathrm{N}$-terminus, while only four (23.5\%) from 17 de novo mutations were located in the N-terminus (Table 1). Regarding four de novo $\mathrm{N}$-terminal mutations, three were at residue 169. In contrast, two maternal mutations (16.7\%) were located in the central domain and two (16.7\%) were located in the C-terminus. One mother carried two mutations in the Central and Cterminus.

In the Ages of parents at birth of probands subsection of the Results, the $\mathrm{P}$ value of the age difference in fathers between de novo and paternal is incorrectly reported as 0.019 . The correct $P$ value is 0.037 .

There are errors in Table 1 and Table 2. Please see the correct tables here.

There are errors in Fig 1 and Fig 3. Please see the correct figures here. f open AcCess

Citation: Ohno S, Hasegawa K, Horie M (2021) Correction: Gender Differences in the Inheritance Mode of RYR2 Mutations in Catecholaminergic Polymorphic Ventricular Tachycardia Patients. PLOS ONE 16(2): e0243476. https://doi.org/ 10.1371/journal.pone.0243476

Published: February 19, 2021

Copyright: $\odot 2021$ Ohno et al. This is an open access article distributed under the terms of the Creative Commons Attribution License, which permits unrestricted use, distribution, and reproduction in any medium, provided the original author and source are credited. 
Table 1. Clinical and genetic summaries of probands.

\begin{tabular}{|c|c|c|c|c|c|c|c|c|c|c|c|}
\hline \multirow{2}{*}{$\begin{array}{l}\text { Patient } \\
\text { Number }\end{array}$} & \multirow[t]{2}{*}{ Sex } & \multicolumn{2}{|l|}{ Age } & \multirow{2}{*}{$\begin{array}{l}\text { Most severe } \\
\text { symptom }\end{array}$} & \multicolumn{3}{|l|}{ RYR2 mutation } & \multirow{2}{*}{$\begin{array}{l}\text { Genotyped Family } \\
\text { Members }\end{array}$} & \multirow[t]{2}{*}{ Inheritance } & \multicolumn{2}{|c|}{$\begin{array}{l}\text { Phenotype of } \\
\text { parents }\end{array}$} \\
\hline & & $\begin{array}{l}\text { Genetic } \\
\text { Analysis }\end{array}$ & Onset & & Nucleotide & Amino Acids & Location & & & \begin{tabular}{|l|} 
parents \\
Father
\end{tabular} & Mother \\
\hline 1 & $\mathrm{~F}$ & 17 & 16 & syncope & exon 3 deletion & N57_G91del35 & NT & Trio & Maternal & none & AF \\
\hline 2 & $\mathrm{~F}$ & 11 & 9 & syncope & exon 3 deletion & N57_G91del35 & NT & P-M & Maternal & none & syncope \\
\hline 3 & $\mathrm{~F}$ & 9 & 9 & syncope & $506 \mathrm{~g}>\mathrm{t}$ & R169L & NT & Trio & de novo & none & none \\
\hline 4 & $\mathrm{~F}$ & 5 & 5 & $\mathrm{CPA}$ & $506 \mathrm{~g}>\mathrm{a}$ & R169Q & NT & Trio & de novo & none & none \\
\hline 5 & $\mathrm{~F}$ & 9 & 8 & $\mathrm{CPA}$ & $506 \mathrm{~g}>\mathrm{a}$ & R169Q & NT & Trio & de novo & none & none \\
\hline 6 & M & 16 & 12 & $\mathrm{CPA}$ & $533 \mathrm{~g}>\mathrm{c}$ & G178A & NT & Trio & de novo & none & none \\
\hline 7 & M & 13 & 11 & syncope & $1221 a>t$ & R407S & NT & P-M & de novo or F & none & none \\
\hline 8 & $\mathrm{~F}$ & 12 & 7 & $\mathrm{CPA}$ & $1259 \mathrm{~g}>\mathrm{a}$ & $\mathrm{R} 420 \mathrm{Q}$ & NT & P-M & Maternal & none & syncope \\
\hline 9 & M & 3 & 3 & syncope & $3667 a>g$ & $\mathrm{~T} 1223 \mathrm{~A}$ & NT & Trio & Maternal & none & none \\
\hline 10 & $\mathrm{~F}$ & 11 & 5 & syncope & $3766 c>a$ & P1256T & NT & Trio & Maternal & none & none \\
\hline 11 & $\mathrm{~F}$ & 15 & 12 & syncope & $4552 c>t$ & L1518F & NT & Trio & Maternal & none & none \\
\hline 12 & $\mathrm{~F}$ & 25 & 10 & syncope & $5170 \mathrm{~g}>\mathrm{a}$ & E1724K & NT & P-M & Maternal & none & syncope \\
\hline 13 & $\mathrm{M}$ & 13 & 13 & $\mathrm{CPA}$ & $6574 a>t$ & M2192L & Central & Trio & Maternal & none & none \\
\hline 14 & $\mathrm{M}$ & 13 & 13 & $\mathrm{CPA}$ & $6737 c>t$ & S2246L & Central & Trio & de novo & none & none \\
\hline 15 & $\mathrm{M}$ & 14 & 11 & syncope & $7024 \mathrm{~g}>\mathrm{a}$ & G2342R & Central & Trio & $\begin{array}{l}\text { Paternal } \\
\text { (mosaic) }\end{array}$ & none & none \\
\hline 16 & $\mathrm{M}$ & 11 & 10 & $\mathrm{CPA}$ & $7169 c>t$ & T2390I & Central & Trio & Paternal & none & none \\
\hline 17 & M & 15 & 10 & $\mathrm{CPA}$ & $7199 \mathrm{~g}>\mathrm{t}$ & $\mathrm{G} 2400 \mathrm{~V}$ & Central & Trio & de novo & none & none \\
\hline 18 & M & 12 & 12 & $\mathrm{CPA}$ & $7423 \mathrm{~g}>\mathrm{t}$ & V2475F & Central & P-M & de novo or $\mathrm{P}$ & none & none \\
\hline 19 & $\mathrm{~F}$ & 18 & 8 & $\mathrm{CPA}$ & $11583 \mathrm{~g}>\mathrm{c}$ & Q3861H & Central & Trio & de novo & none & none \\
\hline 20 & $\mathrm{~F}$ & 8 & 8 & syncope & $11583 \mathrm{~g}>\mathrm{t}$ & Q3861H & Central & Trio & de novo & none & none \\
\hline 21 & $\mathrm{~F}$ & 27 & 6 & syncope & $11836 \mathrm{~g}>\mathrm{a}$ & G3946S & Central & P-M & de novo or $\mathrm{P}$ & none & none \\
\hline 22 & $\mathrm{~F}$ & 16 & 6 & syncope & $11836 \mathrm{~g}>\mathrm{a}$ & G3946S & Central & Trio & de novo & none & none \\
\hline 23 & $\mathrm{~F}$ & 28 & 28 & $\mathrm{CPA}$ & $11917 \mathrm{~g}>\mathrm{a}$ & D3973N & Central & Trio & Maternal & none & none \\
\hline 24 & $\mathrm{M}$ & 3 & 3 & syncope & $12006 \mathrm{~g}>\mathrm{a}$ & M4002I & Central & Trio & de novo & none & none \\
\hline 25 & $\mathrm{M}$ & 11 & 9 & syncope & $12371 \mathrm{~g}>\mathrm{a}$ & S4124N & CT & P-M & Maternal & none & none \\
\hline 26 & $\mathrm{M}$ & 11 & 11 & $\mathrm{CPA}$ & $12458 \mathrm{~g}>\mathrm{t}$ & S4153I & $\mathrm{CT}$ & P-F & de novo or M & none & SD \\
\hline 27 & $\mathrm{M}$ & 11 & 2 & syncope & $12533 a>g$ & N4178S & $\mathrm{CT}$ & Trio & de novo & none & none \\
\hline 28 & $\mathrm{~F}$ & 6 & 6 & $\mathrm{CPA}$ & $12579 c>g$ & C4193W & $\mathrm{CT}$ & Trio & de novo & none & none \\
\hline 29 & M & 10 & 10 & syncope & $13463 a>c$ & Q4488P & CT & Trio & de novo & none & none \\
\hline 30 & $\mathrm{~F}$ & 33 & 9 & syncope & $13798 \mathrm{t}>\mathrm{c}$ & F4600L & $\mathrm{CT}$ & Trio & de novo & none & none \\
\hline 31 & M & 28 & 10 & syncope & $14174 \mathrm{a}>\mathrm{g}$ & $\mathrm{Y} 4725 \mathrm{C}$ & $\mathrm{CT}$ & Trio & de novo & none & none \\
\hline 32 & $\mathrm{~F}$ & 23 & 9 & syncope & $14311 \mathrm{~g}>\mathrm{a}$ & V4771I & $\mathrm{CT}$ & P-M & Maternal & none & syncope \\
\hline 33 & M & 13 & 13 & $\mathrm{CPA}$ & $14311 \mathrm{~g}>\mathrm{a}$ & V4771I & $\mathrm{CT}$ & P-M & de novo or $P$ & none & none \\
\hline 34 & $\mathrm{M}$ & 17 & 14 & $\mathrm{CPA}$ & $14806 c>a$ & Q4936K & $\mathrm{CT}$ & Trio & de novo & none & none \\
\hline 35 & $\mathrm{M}$ & 5 & 5 & $\mathrm{CPA}$ & 14834_14835insTCA & 4944_4945insH & $\mathrm{CT}$ & Trio & de novo & none & none \\
\hline 36 & M & 12 & 12 & СРA & $9910 c>g, 14222 c>t$ & $\begin{array}{l}\text { Q3304E, } \\
\text { A4741V }\end{array}$ & $\begin{array}{l}\text { Central and } \\
\text { CT }\end{array}$ & Trio & Maternal & none & syncope \\
\hline
\end{tabular}

CPA; cardiac pulmonary arrest, NT; N-terminal, CT; C-terminal, SD; sudden death

Table 2. Clinical characteristics of probands with de novo or maternal mutations.

\begin{tabular}{l|l|l}
\hline & de novo & Maternal \\
\hline & $\mathbf{n}=\mathbf{1 7}$ & $\mathbf{n = 1 2}$ \\
\hline Male n (\%) & $9(52.9)$ & $4(33.3)$ \\
\hline Mean age of Onset & $8.1 \pm 3.3$ & $11.0 \pm 6.4$ \\
\hline CPA n (\%) & $9(52.9)$ & $4(33.3)$ \\
\hline Syncope $n$ (\%) & $8(47.1)$ & $8(66.7)$ \\
\hline
\end{tabular}

https://doi.org/10.1371/journal.pone.0243476.t002 


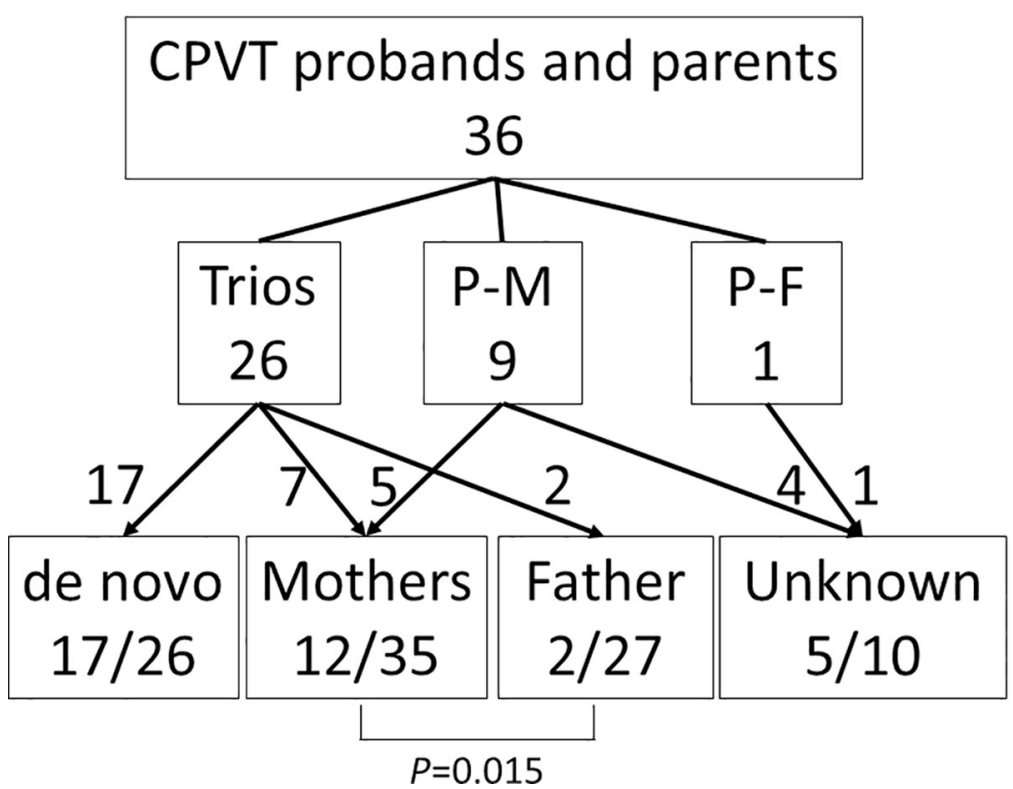

Fig 1. Scheme for Mutation Inheritance. Showing the number of screened family members and the origin of RYR2 mutations. The boxes in the middle lane show genotyped family members in each group. Trio; proband and both parents, P-M; proband and mother, P-F; Proband and father.

https://doi.org/10.1371/journal.pone.0243476.g001

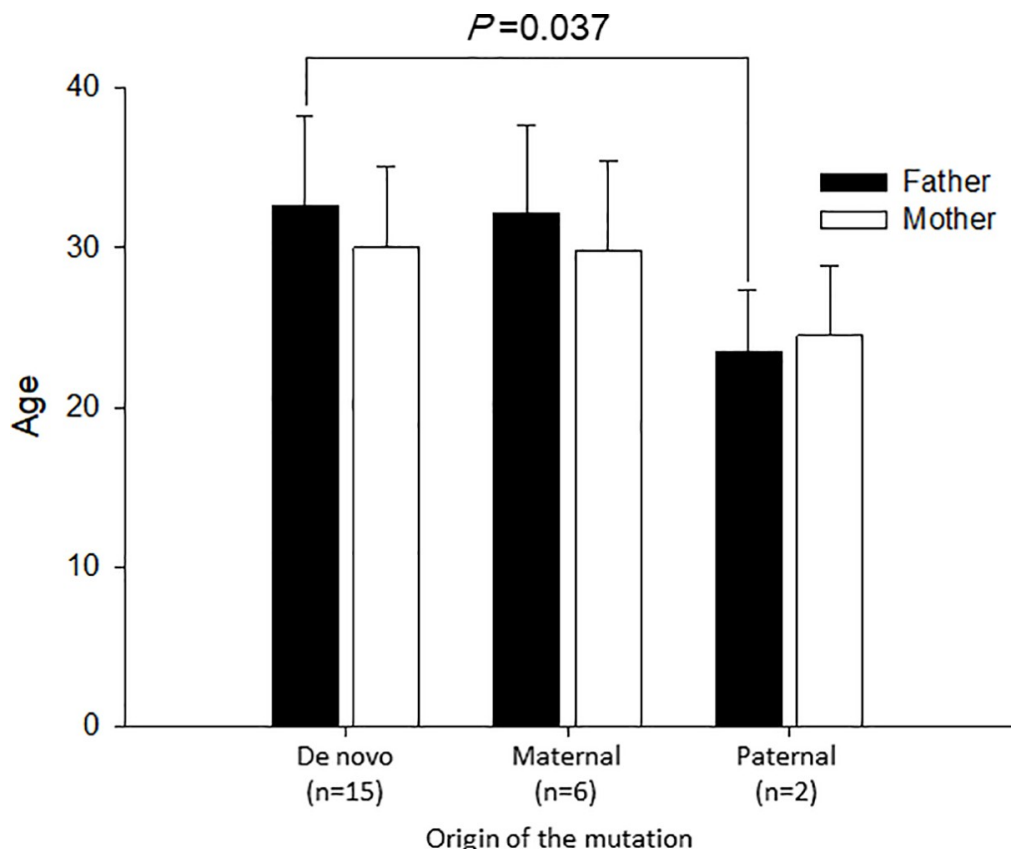

Fig 3. Mean Age of parents depends on the RYR2 mutation origin. Bar graphs depict mean ages of parents at the birth of probands. Filled bars indicate those of fathers and open bars those of mothers. The mean age of genotypepositive fathers was significantly younger than that of the de novo mutation group.

https://doi.org/10.1371/journal.pone.0243476.g002

\section{Reference}

1. Ohno S, Hasegawa K, Horie M (2015) Gender Differences in the Inheritance Mode of RYR2 Mutations in Catecholaminergic Polymorphic Ventricular Tachycardia Patients. PLoS ONE 10(6): e0131517. https://doi.org/10.1371/journal.pone.0131517 PMID: 26114861 